Bull. Austral. Math. Soc.

05в $30,05 \mathrm{~B} 40,05 \mathrm{c} 70$

VOL. 73 (2006) [319-320]

\title{
Small graph designs and their various properties
}

\author{
KAREN G. HARRIS
}

A great deal of work on graph decomposition problems related to 4-cycles has appeared in the literature. Since a 4-cycle is a bipartite graph, this in some sense is the easiest 2-regular graph to consider. In this thesis we branch away from consideration of such a 2-regular graph, but we retain the bipartite property, and we retain a 4-cycle as a subgraph.

The particular graphs considered in this thesis are simple and connected, with six vertices and six edges. It is straightforward to check that there are four such graphs; we refer to them individually as $A, E, S$ and $T$, and collectively as the generic graph $\mathcal{G}$.

In Chapter 2 we find necessary and sufficient conditions for the existence of all $\lambda$ fold $\mathcal{G}$-designs. The problem of decomposing the complete graph, $K_{n}$, into $\alpha$ copies of 4-cycles and $\beta$ copies of $A$ is solved for all possible values of $\alpha$ and $\beta$; this work appears in Chapter 2. Then in Chapter 3 we also deal with those values of $n$ for which $K_{n}$ minus one edge have a decomposition into $\alpha$ 4-cycles and $\beta$ copies of $A$.

For a $G$-design, the intersection problem asks for which values of $k$ is it possible to find two $G$-designs that have $k$ blocks in common, when both $G$-designs are based on the same element set. In Chapter 4 we achieve all the expected intersections numbers for $\mathcal{G}$-designs of order $n \equiv 0,1,4,9(\bmod 12)$.

Another related area of graph decomposition is that of metamorphoses. The intersection problem provides a link between two $\mathcal{G}$-designs which are on the same element set. Metamorphoses provide a link between two distinct decompositions on the same element set into two simple graphs, where one is a proper subgraph of the other and the blocks in one decomposition are subsets of the blocks in the other decomposition. In Chapter 5 we prove that for all admissible orders of $n$, for each $\lambda$, there exists a $\lambda$-fold $A$-design which has a metamorphosis into a $\lambda$-fold 4-cycle system.

It is also of interest to investigate the maximum number of edge-disjoint copies of $\mathcal{G}$ that can be packed into $K_{n}$ so that the number of unused edges is as small as possible. On the other hand, what is the minimum number of copies of $\mathcal{G}$ needed to cover $K_{n}$, so that the number of edges that occur in more than one copy of $\mathcal{G}$ is as small as possible?

Received 1st December, 2005

Thesis submitted to The University of Queensland, June 2005. Degree approved, October 2005. Supervisor: Associate Professor Elizabeth Billington.

Copyright Clearance Centre, Inc. Serial-fee code: 0004-9727/06 \$A2.00+0.00. 
This is known as a maximum $\mathcal{G}$-packing with a leave, and a minimum $\mathcal{G}$-covering with a padding, respectively. We only consider leaves and paddings which are simple graphs. In Chapter 6 we find maximum $\mathcal{G}$-packings of $K_{n}$ with all the expected leaves. For $n \equiv 2,11$ ( $\bmod 12$ ), we establish a minimum $\mathcal{G}$-covering of $K_{n}$ having a padding for just one of the possible 26 simple graphs having five edges. For all other values of $n$, we find minimum $\mathcal{G}$-coverings of $K_{n}$ with all the expected paddings.

We then investigate $\mathcal{G}$-designs with special properties. A $\mathcal{G}$-design is said to be resolvable if the set of its blocks can be partitioned into sets such that each vertex occurs in exactly one block in each set. It is not possible to have a resolvable $\mathcal{G}$-design; but the situation changes when 2-fold designs are considered. We prove in Chapter 7 that a resolvable 2 -fold $A$-design of order $n \equiv 0(\bmod 6)$ exists. A $\mathcal{G}$-design is said to be almost-resolvable if the set of its blocks can be partitioned into sets such that each vertex except one occurs in exactly one block in each set. Again, it is impossible to have a (1-fold) almost resolvable $\mathcal{G}$-design, but instead we prove that an almost-resolvable 2-fold $A$-design of order $n \equiv 1(\bmod 6)$ exists.

A blocking set is a subset of the vertex set of a $\mathcal{G}$-design which intersects every block in the design and does not contain all the elements in any block in the design. A blocking set is said to be minimal when no proper subset of the blocking set is itself a blocking set. In Chapter 8 we construct $A$-designs that have minimal blocking sets.

Department of Mathematics

The University of Queensland

Queensland 4072

Australia

e-mail: Karen.Harris@qed.qld.gov.au 